\title{
Healthcare Knowledge Management: The Art of the Possible
}

\author{
Syed Sibte Raza Abidi \\ NICHE Research Group, Faculty of Computer Science, Dalhousie University \\ Halifax, B3H 1W5, Canada \\ sraza@cs.dal.ca
}

\begin{abstract}
Healthcare knowledge management is an active, yet not a well characterized research topic. In this chapter, we attempt to characterize healthcare knowledge management and highlight the practical aspects of healthcare knowledge management vis-à-vis knowledge-centric services that aim to improve healthcare delivery and health outcomes. We investigate healthcare knowledge management from various perspectives--such as epistemological, organizational learning, knowledge-theoretic and functional. From an epistemological perspective we elicit the different types of healthcare knowledge and the heterogeneous modalities representing it. From a functional perspective we present a suite of healthcare knowledge management services that aim to assist healthcare stakeholders. From a knowledge-theoretic perspective, we present the frontiers of healthcare knowledge management, in particular for patient management through decision support and care planning via a Semantic Web based healthcare knowledge management framework. We conclude by highlighting the role and future outlook of healthcare knowledge management.
\end{abstract}

Keywords: Healthcare knowledge management.

\section{Preamble: The Need for Managing Healthcare Knowledge}

Healthcare is knowledge-rich; yet healthcare knowledge is largely under-utilized at the point-of-care and point-of-need.

Healthcare is experiencing an exponential growth in the scientific understanding of diseases, treatments and care pathways. As a consequence, healthcare knowledge is in flux - new healthcare knowledge is being generated at a rapid pace and its utilization can profoundly impact patient care and health outcomes. But, this growth of knowledge is not congruent with our ability to effectively disseminate, translate and apply current healthcare knowledge in clinical practice. The state-of-affairs is that the large volume of healthcare knowledge, dispersed across different mediums, is making it extremely difficult for healthcare professionals to be aware of and to apply relevant knowledge to make the 'best' patient care decisions. Patient care decisions should be based on best available knowledge applied in line with point-of-care patient data and compliance with the patient's therapeutic preferences. Recent research has shown that the inability of physicians to access and apply current and relevant knowledge healthcare leads to the delivery of suboptimal care to patients [1]. The US Institute of 
Medicine estimated that around 98,000 patients die each year as a consequence of preventable errors [2]. Likewise, a study of two UK hospitals found that $11 \%$ of admitted patients experienced adverse events of which $48 \%$ of these events were most likely preventable if the right knowledge was applied [3]. The inference drawn from the above studies is that the under-utilization of healthcare knowledge contributes to incorrect clinical decisions, medical errors, sub-optimal utilization of resources and high healthcare delivery costs.

Healthcare knowledge is central to clinical decision making throughout the diagnostic-therapeutic cycle-knowledge is applied to arrive at correct diagnostic decisions and to derive the most effective therapeutic regimes. Clinical decisions are made in a cyclic manner, whereby in each cycle the healthcare professional applies knowledge to validate prior hypothesis and satisfy a few more constraints to get closer to the final decision. In this cyclic decision-making process, healthcare knowledge is dynamically contextualized to interpret the patient's evolving health status, and to derive treatment interventions that will work for a specific patient in a specific healthcare setting. Therefore, the key to successful clinical decision-making is the timely availability of correct and relevant knowledge with respect to the clinical context.

Healthcare knowledge is transformative in nature and potential. It is our contention that the apt and timely utilization of healthcare knowledge can transform healthcare practices to achieve high levels of patient safety, care quality, team-care, patient centeredness, and cost-effectiveness. More so, it is of strategic value to address the issues contributing to the under-utilization of knowledge through concerted, systematic and pragmatic mechanisms to 'manage' available healthcare knowledge. Healthcare knowledge management, both as an emerging research theme and a pragmatic practice, aims to manage healthcare knowledge to address the knowledge gaps inherent within a healthcare system [4].

In this chapter, we aim to describe what is healthcare knowledge management and how it can help improve healthcare delivery and practice. This is achieved by firstly characterizing the definition, objective and function of healthcare knowledge management. Next, we elicit the different types of healthcare knowledge and the heterogeneous modalities of healthcare knowledge. Having gained an understanding of healthcare knowledge, next we will provide a broad functional portfolio of healthcare knowledge management. The functional portfolio of healthcare knowledge management will serve as the prelude to the discussion on the research frontiers of healthcare knowledge management. Finally, we will conclude with remarks about future trends in healthcare knowledge management.

\section{Healthcare Knowledge Management}

Healthcare Knowledge Management (HKM) can be characterized as the systematic creation, modeling, sharing, operationalization and translation of healthcare knowledge to improve the quality of patient care. The goal of HKM is to promote and provide optimal, timely, effective and pragmatic healthcare knowledge to healthcare professionals (and even to patients and individuals) where and when they need it to help them make high quality, well-informed and cost-effective patient care decisions. In practice, HKM is pursuing this goal through the advancement of innovative knowledge-mediated solutions 
and their integration in institutional workflows, to improve the quality, efficiency and efficacy of healthcare delivery system [5].

The HKM approach aims to purport a paradigm shift in our understanding of the reality and utility of healthcare knowledge. The HKM paradigm shift advocates a healthcare delivery system that values healthcare knowledge as a vital resource and strives to translate it into clinical practice in order to improve health outcomes. Interestingly, this paradigm shift is largely driven by the unique demands of different healthcare stakeholders, where each stakeholder manifests a specific knowledge need, usage pattern and expected outcome. For instance, the ask from healthcare professionals is not just for mechanisms to easily access knowledge, rather they are demanding the seamless incorporation of current knowledge in clinical workflows to support decision-making [6]. Likewise, patients seek personalized care maps and care-related education to help them understand and cope with their care trajectory. In this paradigm, healthcare knowledge is not just a resource; rather it is a 'service'.

HKM addresses the knowledge gaps experienced by healthcare stakeholders through: (a) a technical infra-structure--i.e. knowledge management strategies, knowledge representation and organization approaches and knowledge processing methods to develop and deploy a knowledge-centric solution; and (b) an operational info-structure--i.e. operational issues and strategies to help incorporate knowledge management solutions in the clinical workflow. Functionally, the HKM portfolio addresses the following `activities:

(a) capture, represent, model, organize and synthesize the different modalities of healthcare knowledge to realize comprehensive, validated and accessible healthcare knowledge resources.

(b) access, share and disseminate current and case-specific knowledge to healthcare stakeholders in a usable format.

(c) operationalize and utilize healthcare knowledge, within clinical workflows, to provide pragmatic patient care services, such as decision-support and careplanning, at the point-of-care and point-of-need.

Numerous challenges still exist to fully realize the HKM portfolio, especially the development of knowledge-centric services that seamlessly integrate within the clinical workflow. The challenges are at multiple levels, such as technical challenges in the design of generic services that can be contextualized to meet a specific user's need; acceptance challenges concerning the usability of a service by stakeholders; and deployment challenges about how to integrate a service in existing infrastructures.

\subsection{HKM as an Approach}

HKM provides a systematic approach to design and deploy knowledge-centric services. The HKM approach covers a broad range of issues, including:

- Integration of heterogeneous healthcare knowledge resources and modalities ranging from evidence-based publications to problem-based discussions to experience-based insights to observation-based health data.

- Modeling of healthcare processes and workflows in general, and then using the model to represent heterogeneous healthcare operational environments. 
- Understanding the specific needs of a range of healthcare knowledge stakeholders-including healthcare practitioners (physicians, nurses, therapists, etc.), administrators, policy makers, patients, care providers, support groups and community-based healthcare workers. Each stakeholder group exhibits different capabilities, orientations, terminology and expectations.

- Handling the dispersion, and subsequent integration, of knowledge across different individuals, departments and institutions.

- Operating on unique clinical situations (as each patient presents a unique set of problems) that demand a contextualized manipulation of available healthcare knowledge to provide patient-specific interventions.

- Applying the same healthcare knowledge, in an inter-changeable and re-usable manner, to different healthcare delivery contexts to achieve improve outcomes.

- Specifying practical and meaningful outcome measurement metrics that relate the utilization of healthcare knowledge to quality of service.

\subsection{HKM as a Change Agent}

It is our contention that HKM initiatives, both in intention and function, can be deemed as change agents that can change the way healthcare knowledge is valued by healthcare stakeholders. In HKM parlance, the change agents are innovative applications that offer high-quality knowledge-centric services, such as: point-of-care decision support; access to evidence based clinical guidelines and literature (i.e. info-buttons); design of optimal clinical workflows/pathways; sharing and re-using experiential knowledge; collection, integration and presentation of health data, in meaningful forms, with respect to the clinical context to support patient care and health policy decisions; translation of knowledge into site-specific practices; and patient-specific care planning. The apt design and deployment of such services can change the utilization potential and pattern of healthcare knowledge in the care delivery process.

\subsection{HKM as a Strategy}

HKM offers a strategy to ensure the successful uptake of HKM applications so that they can serve as change agents. The steps of our proposed HKM strategy are: (i) educate stakeholders about the value of knowledge and demonstrate to them how the application of knowledge will add value to their respective care role; (ii) understand the local care delivery workflow, the user's priorities and resource constraints to design institution-specific applications; (iii) map out the existing knowledge flows within the institution to identify the opportunities and barriers towards the deployment of HKM solutions; (iv) involve stakeholders in determining their perceived relevance to any knowledge-centric service and identify ways in which they might want to use such services; (v) identify the various knowledge resources and then ensure that stakeholders have efficient, and preferably personalized, access to these knowledge resources; and (vi) design HKM applications/frameworks that contextualize knowledge to meet local know-do gaps.

In conclusion, we argue that HKM is not just about a suite of technology-enabled tools, rather it purports a strategy to translate knowledge into policy and practices. 
The success of HKM, therefore, is predicated on our ability to amicably address the 'know-do gap' in a healthcare setting along both technical and strategic dimensions.

\section{The Nature of Healthcare Knowledge: Its Types and Modalities}

Healthcare knowledge is complex both in form and function [7]. In this section we deal with the form of healthcare knowledge and identify the different types of healthcare knowledge, and the various modalities of healthcare knowledge. Here, it is important to appreciate the distinction between knowledge types and modalities: knowledge type refers to the orientation and domain of knowledge, whereas knowledge modality refers to the representation medium in which the knowledge exists.

Healthcare knowledge is primarily employed to support clinical decision-making that in it is a complex activity because it involves an active interplay between different types of healthcare knowledge. We have identified an assortment of knowledge types that directly contribute to clinical decision-making and care planning:

(a) Patient knowledge entails a clear description of the health status of the patient. Patient knowledge encapsulates medical relationships between the various observations of the patient and the inferences drawn by physicians, both captured and recoded in the medical record, to provide a complete picture of the patient.

(b) Practitioner knowledge is practice-related tacit knowledge withheld by a practitioner and exercised whilst discharging patient care [7]. Practitioner knowledge is acquired through active learning, internship, observations and experiences.

(c) Medical knowledge is the core domain knowledge describing the theories about health and healthcare, healthcare delivery models and processes.

(d) Resource knowledge is the quantification of the care delivery resources and infrastructure available within a healthcare setting. It is important for practitioners to have an up-to-date resource knowledge so that they are aware of what resources-such as medical diagnostic devices and tools, drugs, support staff, nurses, hospital beds, surgical facilities and so on - are available when they are making decisions about diagnostic and treatment interventions.

(e) Process knowledge concerns institution-specific care pathways (or workflows) that determine the stipulated discourse of care for specific medical conditions within a healthcare setting. Process knowledge stipulates the standardized way to treat a patient, whilst addressing pragmatic considerations such as the resources needed to treat the patient as per the care pathway.

(f) Organizational knowledge represents the organizational structure and policies exercised by a healthcare institution. Organizational knowledge entails the information and knowledge flows within the organization-i.e. how does information flow from one source to another, who is required to report to whom, what is the decision-making hierarchy, what is the composition of care teams, what are the roles and responsibilities of different healthcare team members, and how to make and respond to information requests. Organizational knowledge is particularly important when deploying HKM solutions because their successful deployment needs to be congruent with the organizational and process knowledge. 
(g) Relationship knowledge reflects the social capital withheld within an organization, a community of healthcare providers or even individuals. In essence, relationship knowledge entails an understanding of how knowledge seeking and sharing can be effectuated between healthcare professional $[8,9]$. In practice, such knowledge helps in asking the right question to the right person-i.e. knowing who is the domain expert, where to get the right knowledge, who can be approached to seek a solution for a specific problem, and who can provide a critical opinion. Relationship knowledge also entails the communication mechanisms and contacts between multiple departments and institutions for the purposes of patient information sharing.

(h) Measurement knowledge details the metrics, criterion and standards to measure success of a healthcare delivery process/system and the associated health outcomes. Measurement knowledge helps (i) to set meaningful performance, efficiency and safety benchmarks, (ii) to measure things that really matter as opposed to superfluous parameters, (iii) to ask the right research questions, (iv) to understand the results with respect to different healthcare contexts, (v) to intelligently analyze the data. Measurement knowledge helps to ascertain whether the knowledge management solution is achieving the desired results, and what is the knowledge uptake within a healthcare setting via the deployed HKM solution.

The above-mentioned healthcare knowledge types are represented by a spectrum of knowledge modalities, where each knowledge modality may capture one or more knowledge types as a healthcare knowledge artifact. Healthcare knowledge artifacts are objects that allow knowledge to be captured and communicated independently of its holder-for instance documents, healthcare records, knowledge bases, communications between peers (emails, blogs, etc) and care workflows. Knowledge artifacts may either be structured, semi-structured, or unstructured.

We have identified the following healthcare knowledge modalities:

(1) Tacit knowledge of practitioners manifested in terms of their problem-solving skills, judgement and intuition.

(2) Explicit knowledge in terms of evidence-based medical literature, reviews, case studies, clinical practice guidelines and so on.

(3) Clinical experiences (both recorded and observed) and lessons learnt.

(4) Collaborative problem-solving discussions between practitioners.

(5) Operational policies eliciting clinical protocols and care pathways.

(6) Educational resources in terms of medical education content for practitioners and health education content for patients.

(7) Decision support (symbolic) rules obtained from domain experts and/or decision models induced from data, and stored in knowledge-bases.

(8) Social knowledge in terms of a community of practice and their communication patterns, interests and expertise of individual community members.

(9) Data-induced observations derived from clinical observations, diagnostic tests and therapeutic treatments recorded in medical records.

In conclusion, we will like to emphasize that our distinction between and characterization of healthcare knowledge types and modalities helps in (a) understanding both the source and pragmatics of a knowledge type, and (b) modeling the knowledge using the right formalism, thus allowing it to be operationalized for HKM services. 


\section{Spectrum of Healthcare Knowledge Management Services}

HKM provides methodologies and methods to develop task-oriented services targeting the specific needs of different healthcare stakeholders. A HKM service provides the functionality to achieve a specified task or function to address the knowledge gaps inherent within the healthcare delivery process. HKM offer a wide spectrum of services that cover the knowledge needs for the entire continuum of the healthcare delivery process. As much as we are familiar with various data-driven services, it is important to emphasize that a HKM service provides a higher-level of abstraction, at times complementing data-driven activities, by providing both semantic-validity and pragmatic-viability to a healthcare solution. Figure 1, presents a high-level spectrum of HKM services, categorized along different healthcare service types.

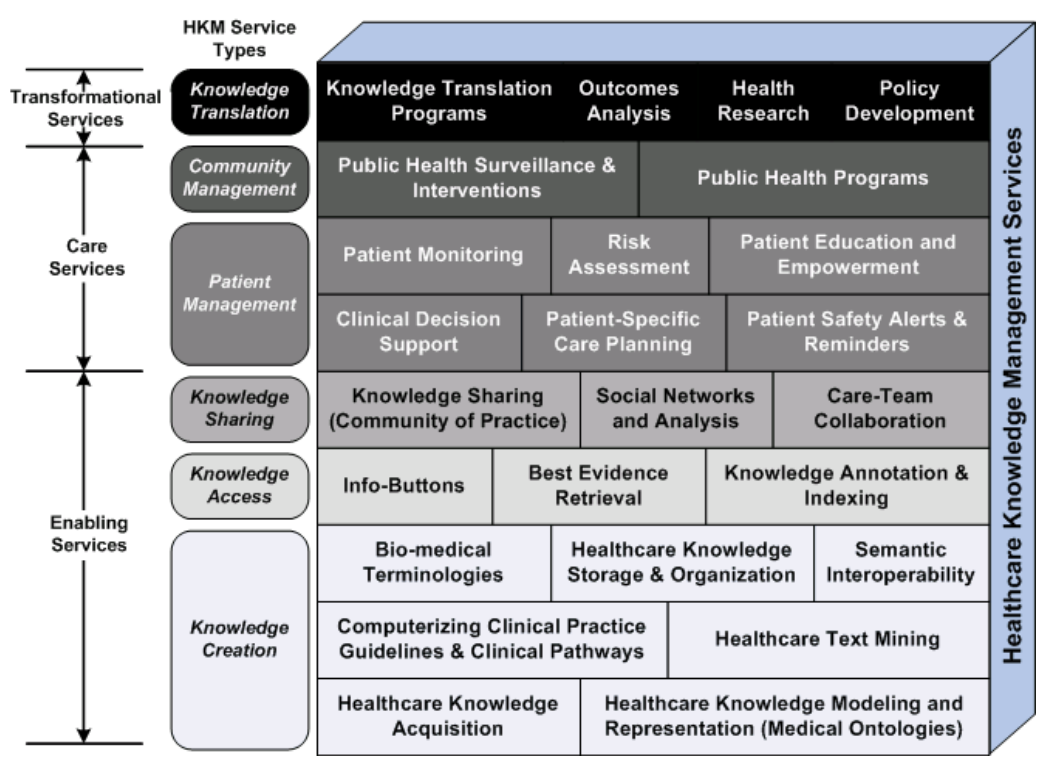

Fig. 1. A hierarchical organization of a spectrum of HKM services

In figure 1, the HKM services are presented as building blocks of a comprehensive and multi-faceted HKM environment that constitutes knowledge creation services as the foundation and knowledge translation services as the tangible outcome. In figure 1, we present a service hierarchy that orders HKM services along the lines of: (a) enabling services that target the identification, collection, organization, modeling of knowledge, in tandem with mechanisms to access the knowledge. Enabling HKM services can be viewed as providing the 'knowledge platform' to develop high-level services; (b) care services that provide the operationalization and utilization of healthcare knowledge. Healthcare stakeholders use care services during the diagnostic-therapeutic cycle to get support for their context-specific healthcare delivery needs. Care services build on the knowledge capital provided by the enabling services, and rely on the transformation services to make them successful; and (c) transformational services that serve as change 
agents to both stipulate and promote a culture of knowledge-centric healthcare practices. Transformational services aim to enhance the uptake of healthcare knowledge through a suite of care services, in order to impact clinical processes, policies, outcome measurements and research. The output of transformational services also serve as a feedback mechanism to guide the design and functionality of the foundational enabling servicesi.e. they provide feedback to streamline the healthcare knowledge resources in line with (i) the needs and demands highlighted by stakeholders; and (ii) the operational barriers and opportunities observed during the use of care services within the healthcare system.

We suggest the novel conceptualization of HKM services as self-contained finegrained 'components' that can be systematically synergized to develop functionally advance HKM applications. Take for instance, the scenario of managing chronic diseases with co-morbidities, which presents severe clinical and operational challenges on the patient, care providers and the healthcare system. The state-of-affairs is that care for chronic diseases with co-morbidities is not fully integrated; individual chronic diseases are managed in specialized units without much awareness of the patient's co-morbidities and the care regime prescribed by different specialized healthcare units. This leads to information gaps about the patient's health, knowledge gaps about how to safely and correctly treat the patient (and not just the disease), and operational gaps leading to duplication of resources allocated through multiple simultaneously active care pathways recommended to the patient. All these gaps not only lead to sub-optimal patient care and compromise patient safety, but they also contribute to the economic burden in treating chronic diseases. In this scenario, we suggest that a combination of HKM services (as listed in Figure 1) may lead to the design of a comprehensive solution for managing patient co-morbidities, as follows: Knowledge creation services will support (i) the acquisition and modelling of relevant healthcare knowledge; (ii) the computerization of clinical practice guidelines and pathways, based on defined knowledge models. The computerization exercise will establish and satisfy clinical pragmatics constraints for merging different clinical practice guidelines and pathways to handle patient's co-morbidities whilst satisfying operational constraints; (iii) the establishment of semantic interoperability between different patient data sources to ensure that patient data for different sources is correctly merged to present a complete patient profile. Knowledge sharing services will provide careteam collaboration mechanisms. Patient management services will support the operationalization of the modelled healthcare knowledge, in conjunction with patient data, to provide decision-support, care-planning, alerts, reminders and patient education services. Knowledge translation services will enable the execution of the care services into practice, whilst providing standard mechanisms to measure the impact of the composite HKM application based on the operational efficiency and clinical efficacy of the care process recommended to patient. The end outcome will be the delivery of high-quality and optimal care for patients with co-morbidities.

We suggest that the design of effective HKM services need to incorporate four interacting dimensions (as shown in figure 2), namely comprehensive and pragmatically sound healthcare knowledge, state-of-the art healthcare knowledge management technology, alignment with institutional clinical workflows, and stakeholder specifications for service needs and usage preferences. The richness of the overlap between these dimensions will determine the functional sophistication of the HKM service. 


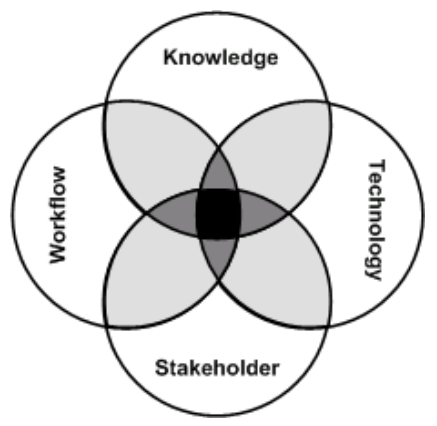

Fig. 2. The four design dimensions of HKM services. The shades of grey highlight the overlap between these dimensions--the darker the shade the more functionally sophisticated is the service. Note that the centre black represents an overlap of all four dimensions, the dark grey an overlap of three dimensions and the light grey an overlap of just two dimensions.

\section{Research Frontiers in Healthcare Knowledge Management}

The research frontiers in HKM are viewed as the attainment of effective, optimal, sound, complete and pragmatic patient management services that are available at the point-of-care and point-of-need. The art-of-the-possible vis-a-vis the next-generation of HKM-based patient management services will be: knowledge-centric, pervasive, pro-active, customized to stakeholder needs, scientific evidence-based, inter-operable between different knowledge sources and operational environments, embedded within clinical workflows, interconnected with patient information systems, compliant to standards, and sensitive to socio-ethical values.

The prevailing patient-centric care delivery paradigm, involving care teams (as opposed to individual care providers) working in concert with the patient, is driving the demand for more sophisticated and broad-based patient management applications. Indeed, this demand is in response to the knowledge-gaps faced by healthcare professionals, who are challenged to provide optimal, safe and high-quality patient management that can be quantifed in terms of improved health outcomes. It is important to note that 'effective and correct' patient management is a challenging activity because it involves a complex, multi-faceted and dynamic interplay between (a) patient parameters that continuously evolve along a temporal axis; (b) up-to-date medical knowledge, existing in different modalities, that needs to be accurately applied during the discourse of the care process; (c) pre-defined clinical pathways that need to be adjusted to meet the patient's conditions; (d) operational constraints defining the healthcare setting; and (e) health outcomes measurement metrics determining the impact of the applied actions. We believe that the next-generation patient management services will extensively leverage healthcare knowledge and HKM strategies to address the above-mentioned patient management challenges.

The emerging Semantic Web framework offers interesting and practical technologies to develop next-generation HKM services. The Semantic Web presents a technical framework to achieve formal semantic modeling-i.e. interpretation, abstraction, axiomatization and annotation - of healthcare knowledge in terms of classes, properties, relations and axioms [10]. The main features of the semantic web framework for HKM 
are: (a) semantic modeling of the procedural and declarative healthcare knowledge as ontologies, which offer a semantically rich and executable knowledge representation formalism; (b) annotation of healthcare knowledge artifacts, guided by the ontological model of the knowledge artifact, to characterize the salient concepts and relations inherent within the artifact. The annotation can be done in terms of the Resource Description Framework (RDF); (c) representation of different patient data sources in a semantically enriched formalism that helps to integrate heterogeneous data sources by establishing semantic similarity between data elements; (d) determining the semantic interoperability between multiple ontologies, using ontology alignment and mediation methods, to dynamically synthesize or morph multiple knowledge resources to address all the facets of a healthcare problem; (e) specifying decision-making logic in terms of symbolic rules, represented as N3 triples, that can be executed using proof engines to infer semanticallysound recommendations/actions; (f) provision of a justification trace of the inferred recommendations to help users understand the rationale for the recommended interventions.

In the next sub-sections, we will highlight some active HKM research trends, and demonstrate our thinking for pursuing these research trends.

\subsection{Patient Longitudinal Care Planning}

Patient care planning support, driven by current and relevant knowledge is highly desirable by healthcare professionals. In this section we present our research effortstowards the development of a lifelong patient management framework, termed CarePlan, to generate adaptive patient-specific care plans that guide a patient's care interventions within a specific healthcare setting [11]. CarePlan offers a knowledgemediated and workflow-oriented solution to patient-centric care planning.

Figure 3 shows the functional design of the CarePlan solution that entails a systematic interplay between various components. The CarePlan activities are initiated with the arrival of a patient to a healthcare setting. The information layer generates a complete profile of the patient by sourcing and integrating patient information from electronic documents, such as electronic health records. The patient's health profile and current health conditions are addressed in a care episode-a temporal combination of episodes constitutes the patient's longitudinal health record. At each episode, the healthcare professional may seek clinical decision and care planning support in terms of a CarePlan query that specifies the care planning requirements to the knowledge layer. The knowledge layer constitutes various knowledge-centric services to derive the knowledge needed to generate a patient-specific CarePlan. First the appropriate CPG are consulted and aligned with the institution's CP to determine the evidence-based and institution-centric knowledge. Next, knowledge morphing is pursued through the dynamic fusion of all relevant knowledge, present in different healthcare knowledge modalities, to generate a comprehensive knowledge object that is passed to the planning layer to generate a CarePlan. The planning layer exploits the morphed knowledge object to design and validate a CarePlan that satisfies all the patient's constraints and is compliant to the operational specifications of the healthcare setting. The planning layer uses proof engines and workflow technologies to generate the CarePlan, which is subsequently validated by a healthcare practitioner. The generated CarePlan is finally passed back to the practitioners. The CarePlan interface allows healthcare practitioners to interact with the CarePlan, which is subsequently stored in the CarePlan database as the patient's longitudinal medical record. 


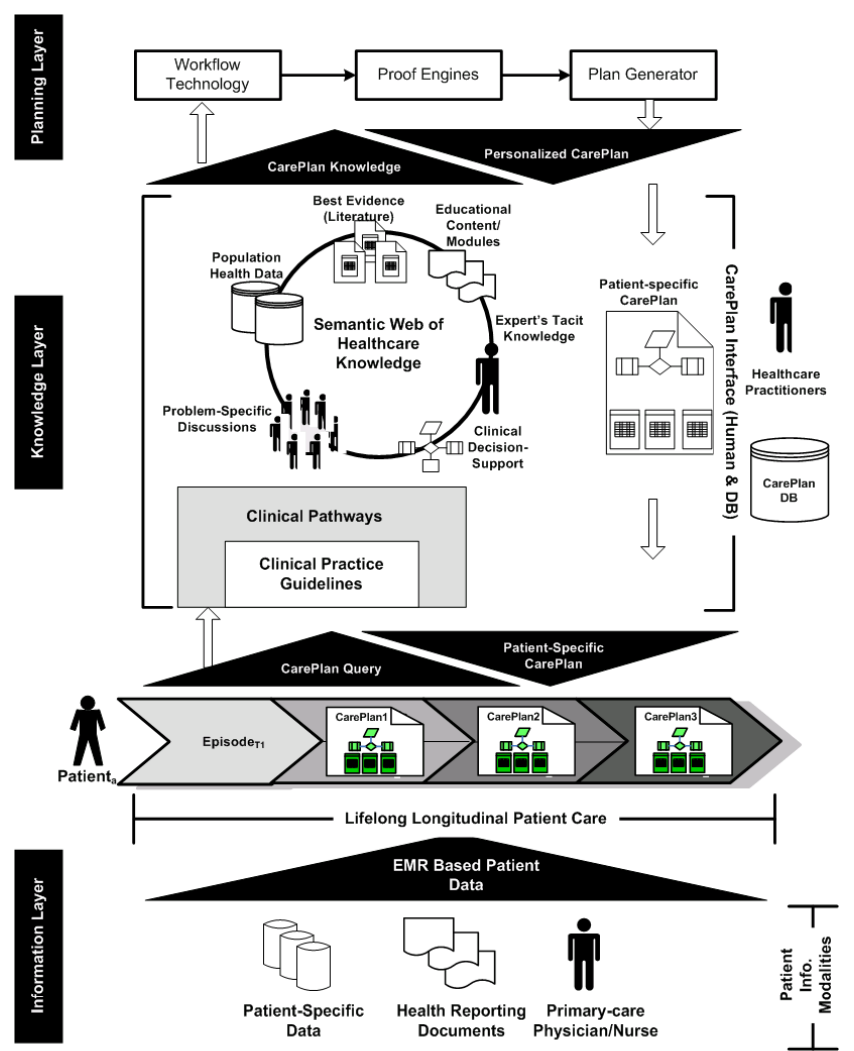

Fig. 3. The functional diagram of the CarePlan framework highlighting the different functional layers and the constituent components

Functionally, CarePlan entails a variety of patient management services (such as clinical decision support, care planning, patient education, alerts and reminders) that realize a personalized care pathway that entails the following: (a) a roadmap of clinical care activities; (b) a recorder of the temporal sequence of medical events, actions and outcomes as they occur in the longitudinal continuum of care. Even variances to the stipulated care plan are recorded; (c) a care-team collaboration medium; (d) a gateway to access case-specific healthcare knowledge to both reason about the patient's diagnosis and to formulate the patient's treatment plan; and (e) patient-specific educational interventions. The feature of CarePlan is that it can handle patients with co-morbidities; it suggests actions and recommendations that are derived after satisfying the clinical pragmatics constraints for the patient's co-morbid conditions. In practice, when a patient with chronic co-morbidities enters the healthcare system, the CarePlan framework will generate a patient-specific CarePlan based on his/her current health profile; and as the patient conditions evolve the associated CarePlan will dynamically adapt to meet the patient's current conditions.

The CarePlan framework is driven by HKM research in the following areas: (i) Ontological modeling of knowledge artifacts, leading to the computerization of clinical 
practice guidelines and clinical pathways; (ii) Medical knowledge morphing; (iii) Ontology-driven decision-support systems; and (iv) Care planning systems based on workflow technology.

\subsection{Knowledge Modeling of Healthcare Knowledge Artifacts}

Clinical Practice Guidelines (CPG) [12] and Clinical Pathways (CP) [13] are evidence-based healthcare knowledge artifacts to assist decision-making and care planning at the point-of-care. CPG are designed to improve outcomes and standardize healthcare. CP basically operationalizes CPG in a specific healthcare setting and serves as a time- and activity-oriented chart for determining and recording the care process.

Lately, there has been a growing interest in harnessing such evidence-based knowledge and translating it into practice. However, most knowledge artifacts are paper-based and hence cannot be translated in practice through computerized clinical applications. Therefore, there is an expansive drive within the HKM research community to computerize knowledge artifacts. The computerization and operationalization of knowledge artifacts is not simple, rather it brings to the forefront several research challenges, such as: (i) abstracting practice-oriented knowledge from the artifact; (ii) modelling and representing the knowledge in a semantically-rich formalism; (iii) incorporating new evidence to the computerized artifact in order to maintain its currency and integrity; (iv) adapting the computerized artifact to meet the operational constraints of the parent institution; (v) customizing the artifact to meet the specific healthcare needs of individual patients; (vi) integrating the computerized artifacts with patient-specific data and clinical applications; and, (vii) executing the computerized artifacts in real-time to provide decision-support and care planning.

Lately, a number of initiatives for healthcare modeling and execution have been reported, such as SAGE [14], GLIF [15], HELEN [16], GUIDE [17], GEM [18] and many others formalisms. The underlying approach of most of these healthcare knowledge modeling formalisms entail the use of ontologies to represent healthcare knowledge. Basically, ontologies provide a formal knowledge modeling method that defines the domain in terms of concepts, relationships and axioms. An ontology-driven modeling approach is highly practical when developing healthcare applications because such applications need to standardize vocabularies and terminologies, establish semantic mappings between multiple patient information databases, describe semantics of the actions, structure knowledge in a potentially executable format and reason over the knowledge. For healthcare knowledge modeling purposes, therefore, ontologies provide both an expressive knowledge representation structure and a logic-based knowledge processing method.

Most of the work in healthcare knowledge modeling focuses on the modeling of $\mathrm{CPG}$ and $\mathrm{CP}$, leading to a various domain-specific ontologies, of varying granularity and efficiency. However, the execution of the knowledge within an ontology, for decision support purposes, based on patient-data is still in its infancy. This is largely due to the lack of sound reasoning engines that can reason over the knowledge represented in ontologies. On the HKM research horizon, there are a number of interesting initiatives that are pursuing both how to operationalize ontology-based knowledge to develop the next-generation Clinical Decision Support Systems (CDSS), and how to 
establish the clinical pragmatics of CDSS in terms of their integration into the clinical workflow of a specific healthcare setting.

From a knowledge modeling perspective, our research focuses on the ontological modeling of CPG and $\mathrm{CP}$, leading to the development of two separate ontologies for CPG and CP. Our research is driven by the research question "how to model the form and function of both CPG and CP, whilst establishing the functional overlap between these knowledge artifacts, in order to computerize and execute them in a clinical setting. The modeling and execution should take into account the potential merging of multiple CPG, multiple CP, and CPG with CP to handle patients with co-morbidities."

Our research approach is grounded in the belief that in order to model CPG and CP we need to understand their underlying components (tasks, actions, decision constructs) and establish the functional relationships between these components. We believe that only after achieving fine-grained, detailed and component level understanding of CPG and CP, we will be able to: (i) distinguish between the functional utility of the various inherent tasks/recommendations, (ii) model the knowledge at a fine-grained level with rich interconnections between multiple components; (iii) merge them in a dynamic and patient-specific manner to handle co-morbidities, (iv) adapt them to satisfy local operational needs and resources, (v) update them efficiently in response to updates to specific knowledge elements, (vi) execute them as a patient-specific care plan, and (vii) incorporate them within clinical workflows.

In line with our approach, we have developed a healthcare knowledge modeling framework, to model both the form and function of CPG [19] and CP [20]. Knowledge modeling entails developing a ontological model of CPG and CP through a reverse-engineering approach whereby we study the actual knowledge artifacts themselves to understand (a) the thought process of the authors of these knowledge artifacts (CPG and CP in this case); (b) the underlying concepts used within these artifacts; and (c) the functional components appearing in the artifacts. This botton-up approach was complemented with a top-down rationalization of the emerging ontology based on the knowledge of the experts involved in the knowledge modeling process. The end outcome was the development of two separate specialized ontologies that represent CPG [19] and CP [20] at a detailed component level, whilst semantically and pragmatically categorizing the inherent knowledge elements and establishing functional and conceptual relationships between the knowledge elements. We present our methodology for healthcare knowledge modeling:

(1) Knowledge Classification: This task involves the overall classification of the knowledge domain along multiple axis in order to assist the selection of a good representative sample of the knowledge artifacts for modeling purposes. We classified CPG along the following six axes: (1) Acute vs. Chronic, (2) Primary vs. Secondary, (3) Specialty Group (Medical vs. Surgical), (4) Setting (Inpatient vs. Outpatient), (5) Age Group, and (6) Orientation (Problem Oriented vs. Task Oriented). The CP were distinguished along five axes: (i) Setting; (ii) Stage of care; (iii) Patient condition; (iv) Intervention; and, (v) Medical specialty.

(2) Knowledge Selection: This task involves the selection of a wide cross-section of knowledge artifacts to build the knowledge model. The selection process is guided by the respective knowledge classification scheme. For our purposes, we selected a mix of 20 different CPG and 30 different $\mathrm{CP}$ from validated resources. 
(3) Knowledge Abstraction: This task involves the abstraction of a knowledge model, in particular the knowledge representation structure of the knowledge artifact. There are two ways of abstracting a knowledge model: (a) acquiring it from domain experts through long and tedious interviews; or (b) studying the knowledge artifact to identify its constituent elements and their relationships. We chose the latter approach. The knowledge abstraction process was guided by the principles of grounded theory, whereby we used a reverse engineering approach to disassemble both CPG and CP into identifiable fine-grained components (such as actions, decisions, recommendations, loops and so on). Next, we analyzed how these components combine and relate with one another to realize a functional CPG or CP. The process involved an iterative analysis, where in each iteration we selected a small number of knowledge artifacts and developed a draft knowledge model that entailed a set of classes, their attributes, relations and constraints. In the next iteration, we selected a few more knowledge artifacts and extended and updated the model with new knowledge found in the current batch of knowledge artifacts. We conducted five such iterations to develop a CPG and CP model that were subsequently used to engineer our CPG and CP ontologies.

(4) Ontology Engineering: This task involved creating the CPG and CP ontology using an ontology engineering process, adapted from the Model-based Incremental Knowledge Engineering (MIKE) process [21]. Ontology engineering comprised cyclical iterations of knowledge acquisition, model design, implementation, and evaluation. The class hierarchy in both ontologies is linked by the class subsumption (is-a) relationship. We used the Protégé-Frames ontology editor and knowledge acquisition system to develop our CPG and CP ontologies.

(5) Ontology Evaluation: This task tests the representational efficiency of the CPG and CP ontologies. We adapted a task-based evaluation strategy [22] that involved the instantiation of five new CPG and CP, using their respective ontologies. During evaluation, we identified ontology deletions (missing concepts), substitutions (ambiguous concepts) and insertions (superfluous concepts).

Our CPG ontology comprises 50 classes (denoted using SMALL CAPS) and 161 attributes (denoted using italics), and we instantiated 15 paper-based CPG. The CPG ontology models the following eight concepts: (i) CPG metadata; (ii) Clinical activities concerning diagnosis and therapy; (iii) Clinical decisions; (iv) Sequential ordering of clinical activities and decisions; (v) Clinical interventions; (vi) Examinations; (vii) Medications; and (viii) Temporal events. The key classes are GuIDELINESTEP to model to different steps in a CPG in terms of ACTION, DECISION and ROUTE subclasses. INTERVENTION_FOR_TREATMENT represents different types of treatment interventions through the following attributes Indication, Contraindication, Criteria_to_check_effects and Action_if_adverse_effects. INTERVENTION_FOR_DIAGNOSIS is defined to represent different diagnostic interventions, and has the following sub-classes: PROCEDURE_ To_Diagnosis, Group_Of_Diagnostic_Processes, PHYSiCAL_EXAM, DiAgnosTIC_IMAGING, LABORATORY_EXAM. To model temporal concepts we defined two classes, named DURATION and SCHEDULE. DURATION has two attributes time_value and time_unit to refer to the time measurement value and the unit respectively. SCHEDULE class has the attributes schedule_type and repetitions to a number to specify the number of schedule repetitions. 
Our CP ontology comprises 141 classes, 230 slots, 1600 instances and 10 constraints, and models 25 CPs. Some of the key classes (denoted using SMALL CAPS) and slots (denoted using italics) are given below. CLEARINGINFORMATION specifies maintenance information for the $\mathrm{CP}$ via slots such as ClinicalPathwayTitle, IntendedAudience, DateDeveloped and ContentSource. TARGETPOPULATION defines the patients for whom the CP is intended, using Age, Sex, InclusionCriteria, and ExclusionCriteria. GOAL describes the overall aim or intention of the CP. SETTING describes the location or environment in which the CP is to be carried out. RoLE indicates the parties accountable for specific tasks, and includes slots that specify the Name, InstitutionalAffiliation, ClinicalPathwayAffiliation, DescriptionOfDuties, and tasks (AccountableFor). PROCESS denotes the larger processes that comprise a CP, where each process has a specified start and end point. Processes may comprise of a series of TASK that specifies the action(s) to be carried out by the healthcare team. Tasks can also be carried out in parallel (TaskConcurrentWith) or in series (TaskFollowedBy). TASK has 14 subclasses that capture task type such as ASSESSMENT, PRESCRIPTION, and DECISION-MAKING. VARIANCE describes deviations from the program of care outlined in the $\mathrm{CP}$, as noted during $\mathrm{CP}$ execution. It includes documentation of VARIANCEDATA, allowing clinicians to describe the nature of the variance.

The complete details of the CPG and CP ontologies cannot be provided here due to space limitations, but these details will be available through future publications.

\subsection{Clinical Decision Support Systems}

CDSS are knowledge-based systems that provide healthcare professionals with patient-specific recommendations and intervention, based on the patient's health status [23]. Clinical interventions provided by CDSS include evidence-based recommendations, alerts, reminders, risk assessments, diagnostic support, clinical workflow, order sets, patient information dashboards and care documentation templates. CDSS are quite effective in improving health outcomes, patient safety and healthcare costs.

The recent trend in CDSS development is to incorporate evidence-based knowledge artifacts--such as CPG, CP--as the source of knowledge [24]. This is a major undertaking as it requires first the computerization of the knowledge artifact and then the incorporation of the computerized knowledge artifact within a reasoning engine to deliver evidence-based recommendations. For developing CDSS based on CPG the key activities are: (i) The computerization of the CPG into an executable format. The computerization exercise needs to capture and represent the disease-specific knowledge inherent within a CPG, whilst maintaining the underlying clinical pragmatics and identifying the key decisional elements; (ii) The specification of a sequence of actions to realize an executable CPG plan. This step demands identifying the causal and temporal relationship between different clinical actions and the outcome of these clinical actions; (iii) The transformation of the CPG decision logic into medically salient and executable logic-based decision rules; (iv) The execution of the CPG decision logic, using logic-based reasoning engines, based on both acquired and inferred patient information; (v) The explanation of the CDSS recommendations in order to establish 'trust' with the user; and (vi) The collection of patient information, either through an interactive user-interface or directly from the electronic medical record, as demanded by the CPG execution engine in order to execute the CPG decision-rules. 
We have developed a Semantic Web based CDSS for helping family physicians discharge breast cancer follow-ups [25, 26]. The CDSS was based on a breast cancer follow-up CPG that we computerized, in order to provide CPG-mediated recommendations for breast-cancer follow-up care. Our CDSS constitutes three key elements:

(a) Modeling the overall declarative and procedural knowledge required for decision support. Our approach was to independently model the different types of knowledge required for decision support and them integrate the knowledge as needed. We identified three knowledge types and developed three independent, yet interacting ontologies: (i) CPG Ontology that models the structure of the CPG. We use the Guideline Element Model (GEM) [18] as the modeling formalism and the CPG ontology is based on the GEM structure; (ii) Domain Ontology that models the medical knowledge pertaining to the domain of breast cancer. The knowledge was derived from the breast cancer CPG being modeled [27]; and (iii) Patient Ontology that models the patient's parameters necessary to execute the CPG. We used Protégé OWL to develop all the ontologies.

(b) Authoring of CPG-medicated decision rules based on the knowledge represented within the three ontologies. For rule authoring purposes the ontologies are aligned at the property level to realize a multi-faceted knowledge-base for the breast cancer follow-up CDSS. Our rule authoring approach ensures that only pre-defined knowledge is used thus ensuring the sanity of the decision rules.

(c) Execution of the CPG to provide case-specific decision support. We developed a CPG execution engine, using JENA-a logic-based proof engine that executes the decision logic rules based on patient data. The CPG execution output is a set of inferred recommendations based on the patient data. To assist family physicians, for each recommendation we provide (a) the CPG-based explanation; (b) references to related literature, and (c) a justification trace showing how a recommendation was derived by the decision rules using the given patient data.

The breast cancer CDSS will be deployed in family-care settings, and is intended to serve both as a decision-support tool and a knowledge translation medium for family physicians who are not specialists in breast-cancer follow-up care.

\subsection{Healthcare Knowledge Morphing}

We know that clinical decision-making is a complex activity that involves an active interplay between various healthcare knowledge modalities. Healthcare practitioners when making clinical decisions are able to (a) 'intrinsically' determine the function and correlation between knowledge objects represented in heterogeneous modalities; (b) mentally morph multiple knowledge resources-i.e. synthesize tacit knowledge with experiential knowledge with explicit knowledge--to form a comprehensive knowledge object to address the problem; and (c) finally operationalize the knowledge object as per the demands of the clinical problem at hand.

We posit that, if CDSS are expected to provide high-quality and pragmatic decision support interventions then the use of a single knowledge modality may not suffice. Rather, what is needed is the seamless morphing of various knowledge modalities to dynamically create a problem-specific holistic knowledge-base that can competently interpret a complex clinical scenario and in turn recommend a set of 
actions that are grounded in both evidence and experience. This demands research in healthcare knowledge morphing that entails "the intelligent and autonomous fusion/integration of contextually, conceptually and functionally related knowledge objects that may exist in different representation modalities and formalisms, in order to establish a comprehensive, multi-faceted and networked view of all knowledge pertaining to a domain-specific problem" [28].

Healthcare knowledge morphing is an interesting, yet challenging, HKM research theme. In our work, we are pursuing healthcare knowledge morphing using a semantic web framework, as shown in figure 4.

The key activities of our knowledge morphing framework are [29]:

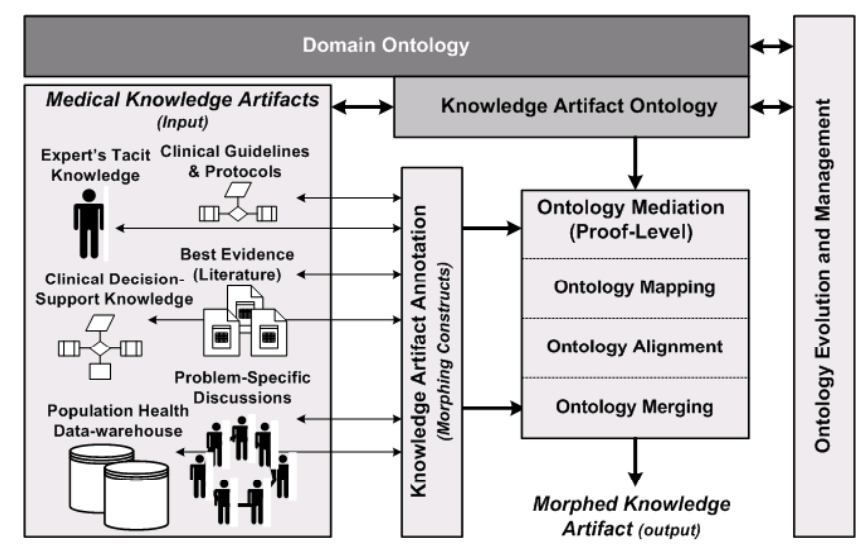

Fig. 4. The functional diagram of our healthcare knowledge morphing framework [29]

(a) Medical knowledge representation: Knowledge representation is achieved through ontologies. For knowledge morphing purposes, we distinguish two different ontologies-(a) a high-level domain ontology that describes the medical concepts of the domain-i.e. the declarative knowledge; and (b) a lower-level knowledge artifact ontology that captures both the structure and content of a particular knowledge artifact, thus representing the procedural knowledge and operational parameters that stipulate the use of the knowledge in a healthcare setting.

(b) Knowledge artifact annotation: The objective of the knowledge annotation activity is to establish morphing constructs within knowledge artifacts to facilitate their morphing with other knowledge artifacts. We use the domain and knowledge artifact ontologies to identify and annotate the possible morphing constructs within candidate knowledge artifacts, in anticipation of morphing them.

(c) Healthcare knowledge morphing: Knowledge morphing is achieved via prooflevel ontology mediation whereby the candidate knowledge artifacts are logically morphed based on contextual and functional congruence determined by proofengines. The process of ontology mediation comprises the activities of Ontology mapping, alignment and merging to yield knowledge morphing.

(d) Ontology evolution and management: This activity is to track any updates to the healthcare knowledge encapsulated within the ontologies, and then incorporating 
these updates to the ontologies. We perform periodic updates to both the domain and knowledge artifact ontologies to ensure that they are complete and valid.

\section{Postscript}

There is a growing demand by healthcare stakeholders for pragmatic, proactive, multi-faceted and comprehensive healthcare knowledge to be available at the pointof-care. This demand by health stakeholders, though reasonable and valid, is not achievable unless we are ready to uptake HKM principles and practices within clinical workflows, and develop the necessary capacity amongst healthcare professionals to manage the knowledge. Indeed, there is a general lack of understanding about the potential of HKM that is resulting in the prevalence of operational barriers towards the flow and use of knowledge within the healthcare system. However, it is our contention that recent advancements in HKM applications will effectively bring down these barriers, because these applications will practically demonstrate how they can effectively help to achieve high levels of patient safety, care quality, team-care, patient centeredness, and cost-effectiveness. Nevertheless, if we want to sustain the growing demand for HKM principles and practices to realize a knowledge-cognizant healthcare system, then it is imperative that we address the scalability, variability and validity issues concerning both healthcare knowledge and knowledge-centric services.

These are exciting times in the evolution of HKM as a discipline and a demonstration of the art-of-the-possible in patient care. Indeed, there are a number of challenges that require us to investigate the technical, operational and strategic aspects of HKMdriven systems. But, it is encouraging to note that the HKM research landscape presents numerous research initiatives that are addressing complex multi-faceted HKM issues, and most promisingly the research outcomes are maturing in terms of a rich offering of practical knowledge-centric applications that are beginning to positively impact patient care in healthcare settings. We believe that more complete and sophisticated HKM solutions will emerge from the synthesis and cross-fertilization of ongoing HKM research themes, ideas and outcomes. Indeed, the future of HKM is challenging, promising and fulfilling.

\section{References}

[1] McGlynn, E.A., Asch, S.M., Adams, J., Keesey, J., Hicks, J.: The Quality of Health Care Delivered to Adults in the United States. N. Engl. J. Med. 348, 2635-2645 (2003)

[2] Kohn, L.T., Corrigan, J.M., Donaldson, M.S. (eds.): To Err is Human: Building a Safer Health System. National Academy Press, Washington, DC (1999)

[3] Vincent, C., Neale, G., Woloshynowych, M.: Adverse Events in British Hospitals: Preliminary Retrospective Record Review. BMJ 322, 517-519 (2001)

[4] Bali, R., Dwivedi, A.: Healthcare Knowledge Management: Issues, Advances and Successes. Springer, Heidelberg (2006)

[5] Jackson, J.R.: The Urgent Call for Knowledge Management in Medicine. Physician Exec. 26, 28-31 (2000)

[6] Montani, S., Bellazzi, R.: Supporting Decisions in Medical Applications: The Knowledge Management Perspective. Intl. Journal of Medical Informatics 68, 79-90 (2002) 
[7] Wyatt, J.: Management of Explicit and Tacit Knowledge. J. Royal. Soc. Med. 94, 6-9 (2001)

[8] Abidi, S.S.R.: Healthcare Knowledge Sharing: Purpose, Practices and Prospects. In: Bali, R.K., Dwivedi, A. (eds.) Healthcare Knowledge Management: Issues, Advances and Successes, pp. 65-86. Springer, Heidelberg (2006)

[9] Curran, J., Abidi, S.S.R.: Evaluation of an Online Discussion Forum for Emergency Practitioners. Health Informatics Journal 13(4), 255-266 (2007)

[10] Berners-Lee, T., Hendler, J., Lassila, O.: The Semantic Web. In: Scientific American (2001)

[11] Abidi, S.S.R., Chen, H.: Adaptable Personalized Care Planning via a Semantic Web Framework. In: 20th Intl Cong European Fed for Medical Informatics Maastricht (2006)

[12] Davis, D., Taylor-Vaisey, A.: Translating Guidelines into Practice: A Systematic Review of Theoretic Concepts, Practical Experience and Research Evidence in the Adoption of Clinical Practice Guidelines. Can. Med. Assoc. 157(4), 408-416 (1997)

[13] de Bleser, L., Depreitere, R., de Waele, K., Vanhaecht, K., Vlayen, J., Sermeus, W.: Defining Pathways. J. Nurs. Manag. 14, 553-563 (2006)

[14] Tu, S.W., Campbell, J.R., Glasgow, J., Nyman, M.A., McClure, R., McClay, J., Parker, C., Hrabak, K.M., Berg, D., Weida, T., Mansfield, J.G., et al.: The SAGE Guideline Model: Achievements and Overview. J. Am. Med. Inform. Assoc. 14(5), 589-598 (2007)

[15] Boxwala, A.A., Peleg, M., Tu, S., Ogunyemi, O., Zeng, Q.T., Wang, D., Patel, V.L., Greenes, R.A., Shortliffe, E.H.: GLIF3: A Representation Format for Sharable Computerinterpretable Clinical Practice Guidelines. J. Biomed. Inform. 37, 147-161 (2004)

[16] Haschler, I., Skonetzki, S., Gausepohl, H.J.: Evolution of the HELEN Representation for Managing Clinical Practice Guidelines. Methods. Inf. Med. 43(4), 413-426 (2004)

[17] Ciccarese, P., Caffi, E., Boiocchi, L., Quaglini, S., Stefanelli, M.: A Guideline Management System. In: Medinfo. 11th World Congress on Medical Informatics (2004)

[18] Shiffman, R.N., Karras, B.T., Agrawal, A., et al.: GEM: A Proposal for a More Comprehensive Guideline Document Model Using XML. JAMIA 7, 488-498 (2000)

[19] Shapoor, S.: Knowledge Modeling to Develop a Clinical Practice Guideline Ontology: Towards Computerization and Merging of Clinical Practice Guidelines. Masters Thesis. Dalhousie University (2007)

[20] Hurley, K., Abidi, S.S.R.: Ontology Engineering to Model Clinical Pathways: Towards the Computerization and Execution of Clinical Pathways. In: 20th IEEE Symposium on Computer-Based Medical Systems, Maribor, IEEE Press, Los Alamitos (2007)

[21] Angele, J., Fensel, D., Studer, R.: Domain and Task Modeling in MIKE. In: Proceedings of IFIP WG 8.1/13.2 Joint Working Conference, pp. 149-163 (1996)

[22] Porzel, R., Malaka, R.: A Task-based Approach for Ontology Evaluation. In: ECAI Workshop on Ontology Learning and Population, Valencia (2004)

[23] Sim, I., Gorman, P., Greenes, R.A., Haynes, R.B.: Clinical Decision Support Systems for the Practice of Evidence-based Medicine. J. Am. Med. Inform. Assoc. 8, 527-534 (2001)

[24] Bates, G.J., Kuperman, S., Wang, T., Gandhi, A., Kittler, L.: Ten Commandments for Effective Clinical Decision Support: Making the Practice of Evidence-based Medicine a Reality. J. Am. Med. Inform. Assoc. 10, 523-530 (2003)

[25] Abidi, S., Abidi, S.S.R., Hussain, S., Shepherd, M.: Ontology-based Modelling of Clinical Practice Guidelines: A Clinical Decision Support System for Breast Cancer Follow-up Interventions at Primary Care Settings. In: 12th World Congress on Medical Informatics (2007) 
[26] Hussain, S., Abidi, S., Abidi, S.S.R.: Semantic Web Framework for Knowledge-Centric Clinical Decision Support Systems. In: 11th Conference on Artificial Intelligence in Medicine, Amsterdam, Springer, Heidelberg (2007)

[27] Abidi, S.: Ontology-based Modeling of Breast Cancer Follow-up Clinical Practice Guideline for Providing Clinical Decision Support. In: 20th IEEE Symposium on ComputerBased Medical Systems, Maribor, IEEE Press, Los Alamitos (2007)

[28] Abidi, S.S.R.: Medical Knowledge Morphing: Towards Case-Specific Integration of Heterogeneous Medical Knowledge Resources. In: 18th IEEE International Symposium on Computer-Based Medical Systems, Dublin, IEEE Press, Los Alamitos (2005)

[29] Abidi, S.S.R., Hussain, S.: Medical Knowledge Morphing via a Semantic Web Framework. In: 20th IEEE Symposium on Computer-Based Medical Systems, Maribor, IEEE Press, Los Alamitos (2007) 